\title{
Gesprächsdatenbanken als methodisches Instrument der Interaktionalen Linguistik - Eine exemplarische Untersuchung auf Basis des Korpus FOLK in der Datenbank für Gesprochenes Deutsch (DGD2)
}

\author{
Arnulf Deppermann / Thomas Schmidt
}

\section{Was ist ein Gesprächskorpus?}

Unter einem Gesprächskorpus verstehen wir eine Sammlung von Aufzeichnungen (Audio- und/oder Videoaufnahmen) authentischer Gespräche (i.e. konzeptionell und medial mündlicher, i.d. R. spontaner, Interaktion von zwei oder mehr Teilnehmern), die nach einer wissenschaftlich begründeten und explizit dargelegten Systematik zusammengestellt und über eine Transkription, ggf. zusätzliche Annotationen und die Dokumentation von Metadaten (zu Gesprächsumständen und beteiligten Sprechern) für eine (sprach-)wissenschaftliche Analyse erschlossen wird. Gesprächskorpora sind zum einen abzugrenzen von schriftsprachlichen Korpora, bei denen die Analyse unmittelbar auf den Primärdaten (i.e. den schriftsprachlichen Texten) aufsetzt, ohne auf die Erschließung mittels theoriegeleiteter Abstraktionen wie Transkriptionen angewiesen zu sein. Zum anderen sind sie zu unterscheiden von anderen Typen mïndlicher Korpora, die Gespräche beinhalten, welche eigens für Forschungszwecke geführt wurden, d. h. Interviewkorpora, die der Bearbeitung soziolinguistischer (z. B. Labov 1972) oder dialektologischer (z.B. Zwirner-Korpus, Bethge/Bonnin 1969) Fragestellungen dienen, oder sog. $\gg$ Speech Corpora zur Konstruktion sprachtechnologischer Anwendungen (z.B. Hinrichs et al. 2000). Neben FOLK existieren für das Deutsche weitere Gesprächskorpora - beispielsweise das "Freiburger Korpus « (Steger/Engel/Moser 1971), das Korpus »Dialogstrukturen (Berens et al 1976, beide ebenfalls über die DGD2 zugänglich), das Korpus »Gesprochene Wissenschaftssprache Kontrastiv« (GeWiss, Fandrych et al. 2012) oder das »Kiezdeutsch «-Korpus (KidKo, Wiese 2012).

Gesprächskorpora können die Analysegrundlage für sehr unterschiedliche Fragestellungen aus verschiedenen Teilgebieten der Linguistik bilden - beispielweise ermöglichen sie einem Lexikographen eine korpusbasierte Untersuchung des mündlichen Gebrauchs eines Lexems, einem Phonetiker (eine ausreichende Aufnalımequalität vorausgesetzt) lautliche Analysen von Spontansprache oder einem Computerlinguisten die Studie von Dialogstrukturen als Grundlage von Systemen zur automatischen Sprachverarbeitung. Das zentrale Anwendungsfeld für Gesprächskorpora sind aber sicherlich diejenigen Teilgebiete der Linguistik, die die Gesprächsstrukturen und -praktiken und die Verwendung sprachlicher Formen in der sozialen Interaktion zum zentralen For- 
Gesprächsdatenbanken als methodisches Instrument der Interaktionalen Linguistik

schungsgegenstand haben, insbesondere also verschiedene Ausprägungen der Gesprächsforschung wie die Konversationsanalyse, die interaktionale Linguistik, die funktionale Pragmatik oder die Gesprochene-Sprache-Forschung.

\section{Das Forschungs- und Lehrkorpus Gesprochenes Deutsch (FOLK)}

\begin{tabular}{|l|l|}
\hline Ort & Region: Rheinfränkische Sprachregion \\
\hline $\begin{array}{l}\text { Institution / Räumlichkei- } \\
\text { ten }\end{array}$ & Nicht vorhanden / Wohnzimmer \\
\hline Aufnahmebedingungen & Pokerchips machen Geräusche auf dem Tisch \\
\hline 3 dokumentierte Sprecher & $\begin{array}{l}\text { FOLK_S_00054 } \\
\text { (Mitspicler in FOLK_E_00040_SE_01) } \\
\text { FOLK_S_00055 } \\
\text { (Mitspieler in FOLK_E_00040_SE_01) } \\
\text { FOLK__00056 } \\
\text { (Mitspieler in FOLK_E_00040_SE_01) }\end{array}$ \\
\hline
\end{tabular}

Abbildung 1: Ereignismetadaten in FOLK (Ausschnitt)

Das »Forschungs- und Lehrkorpus Gesprochenes Deutsch« (FOLK) wird seit 2008 im Archiv für Gesprochenes Deutsch am IDS Mannheim aufgebaut. Es ist ein Gesprächskorpus mit den folgenden Eigenschaften:

- es beinhaltet Aufnahmen ganzer Gespräche;

- Metadateninformationen über Gesprächsereignisse und Sprecher werden nach einem einheitlichen Schema dokumentiert (siehe Abbildung 1);

- die Gesprächsaufnahmen werden vollständig in literarischer Umschrift nach den Vorgaben des GAT2-Systems (Selting et al. 2009) für Minimaltranskripte mit dem Editor FOLKER (Schmidt/Schitte 2010) transkribiert, Aufnahmen und Transkripte werden dabei in Segmenten von etwa 3 bis 5 Sekunden Länge miteinander aligniert;

- zusätzlich zur literarischen Umschrift erfolgen eine standardorthographische Normalisierung der transkribierten Formen, sowie eine Lemmatisierung und ein Part-Of-Speech-Tagging auf zusätzlichen Annotationsebenen (siehe Abbildung 2 und Westpfahl/Schmidt 2013). 
Arnulf Deppermann / Thomas Schmidt

\begin{tabular}{|l|l|l|l|l|l|l|l|l|}
\hline Transkription & da & gehst & de & jetz & einfach & über & dern & bild \\
\hline Normalisierung & da & gehst & du & jetzt & einfach & über & dem & Bil \\
\hline Lemmatisierun & da & gehen & du & jetzt & einfach & über & d & Bil \\
\hline POS & ADV & VFIN & PPER & ADV & ADJD & APPR & ART & NN \\
\hline
\end{tabular}

Abbildung 2: Annotationsebenen in FOLK

FOLK ist als dauerhaft wachsendes Korpus konzipiert, d.h. es soll in den kommenden Jahren kontinuierlich um neue Aufnahmen ergänzt werden. Die Strategie zur Auswahl von Gesprächen in der ersten Ausbauphase beschreiben Deppermann/Hartung (2011, $445 \mathrm{f}$.) wie folgt:

"Priorität für die Korpuserstellung ist es dabei zunächst, die grundlegenden Parameter der >Merkmale der Sprechsituation $[$ [...] möglichst breit zu variieren. Das erste Ziel besteht darin, eine möglichst breite Spannweite unterschiedlicher kommunikativer Anlässe und Formen (Gattungen) im Korpus abzudecken.»

Diese erste Ausbauphase ist mit der Veröffentlichung von ca. $100 \mathrm{~h}$ Aufnahmen bzw. ca. 1 Millionen transkribierter Wort-Tokens Anfang 2014 abgeschlossen. Die bereits verfügbaren Daten umfassen eine breite Spannweite von unterschiedlichen Typen von Alltagskommunikation (z. B. Tischgespräche, Spielinteraktionen zwischen Erwachsenen, verschiedene Formen der Eltern/Kind-Interaktion) und institutioneller Kommunikation (z. B. Unterrichtskommunikation, Prüfungsgespräche, verschiedene Formen beruflicher Kommunikation), dazu kommen Aufnahmen öffentlich-medialer Kommunikation (Schlichtungsgespräche zu Stuttgart 21). Weiteres Ziel für die zukünftige Korpuserweiterung ist eine stärkere regionale und altersbezogene Variationsbreite des Korpus, in dem zur Zeit Aufnahmen von Sprechern mit höherem Bildungsabschluss im Alter zwischen 20 und 30 aus der rheinfränkischen Sprachregion dominieren.

Ziel kann dabei allerdings nicht sein, ein vollständig ausgewogenes Korpus zu erstellen. Dafür ist die Zahl der interessierenden Variablen, bezüglich đerer eine Ausgewogenheit herzustellen wäre, zu groß. Eine ausgewogene Stratifizierung, beispielsweise nach Ort der Erhebung, Alter, Geschlecht und Bildungsstand der Sprecher, die zusätzlich auch noch eine grobe Gesprächstypenklassifuzierung mit einbezieht (etwa Alltags- vs. institutionelle Kommunikation), würde bedeuten, dass für jede Kombination von Variablenausprägungen (bspw. > Alltagsgespräche aus dem bairischen Sprachraum mit ailteren männlichen Sprechern mit niedrigerem Bildungsabschluss) ausreichend und gleich große Datenmengen in das Korpus einfließen müssten. Dies wäre angesichts des Aufwandes, der mit der Datenerhebung und -aufbereitung verbunden ist, eine utopische Anforderung. Statt also die Variablen in Kombination zu betrachten, soll daher künftig versucht 
Gesprächsdatenbanken als methodisches Instrument der Interaktionalen Linguistik

werden, zumindest zu jeder einzelnen Variablen-Ausprägung ausreichend (nicht aber unbedingt gleiche Mengen an) Daten im Korpus zu haben - also etwa bei den Erhebungsorten keine sprachliche Großregion auszulassen, und Sprecher aller Altersklassen und Bildungsstufen zu berilcksichtigen. Damit wird zwar keine Ausgewogenheit des Korpus als Ganzes erreicht, es ist aber immerhin möglich, aus dem Gesamtbestand Teilkorpora zu bilden, die bezigglich einer ausgewählten Variablen ausgewogen sind. Die technische Voraussetzung hierfür ist eine Funktionalität, die es dem Benutzer erlaubt, durch systematische Suchanfragen auf Metadaten und/oder manuelle Auswahl einzelner Gesprächsereignisse virtuelle Korpora zu bilden und die weitere Analyse dann auf ein solches virtuelles Korpus zu beschränken. Die DGD2 stellt eine solche Funktionalität bereits zur Verfügung (siehe Abbildung 3).

\begin{tabular}{|c|c|c|c|c|c|}
\hline$\sqrt{v}$ & 100 & FOLK_E_00189 & Nordnlederdeutsche Sprachregion & FOLK_S_60431 & Mănnlich \\
\hline$\sqrt{2}$ & 2.09 & FOLKE 00100 & Nordniederdeutsche Sprachregion & FOLK S 00301 & Mannilica \\
\hline$\sqrt{18}$ & $\begin{array}{ll}3 & 0,0\end{array}$ & FOLK_E_00107 & Nordniederdeutsche Sprachregion & FOLK_S_00276 & Mannlich \\
\hline$\nabla$ & 4,10 & FOLK_E_00178 & Bairische Sprachreglon & $\begin{array}{l}\text { FOLKS } 90420 \\
\text { FOLK S } 00433\end{array}$ & Aannlich \\
\hline 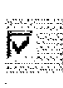 & 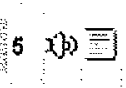 & FOLK_E_00186 & Balrische Sprachregion & $\begin{array}{l}\text { FOLK_S_00426 } \\
\text { FOLK_S_00.433 }\end{array}$ & $\begin{array}{l}\text { Männlich } \\
\text { Mãnnlich }\end{array}$ \\
\hline$\sqrt{2}$ & 6 & FOLK_E_00184 & Nordnlederdeutsche Sprachregion & $\begin{array}{l}\text { HOLK S } 00424 \\
\text { FOLK S 00432 }\end{array}$ & $\begin{array}{l}\text { Männlich } \\
\text { Mánnlich }\end{array}$ \\
\hline
\end{tabular}

Abbildung 3: Zusammenstellen eines virtuellen Korpus (Männliche Sprecher aus drei ausgewählten Sprachregionen)

\section{Gesprächsdatenbanken: Die Datenbank für Gesprochenes Deutsch (DGD2)}

Außer durch die Daten selbst werđen die Nutzungsmöglichkeiten eines Gesprächskorpus ganz wesentlich mitbestimmt durch die Instrumente, die für deren Analyse zur Verfügung stehen. Erst durch eine geeignete informationstechnische Erschließ3ung der Korpusdaten können diese ihr volles Potential entfalten. Das zentrale informationstechnologische Instrument für die Nutzung von FOLK ist die Datenbank für Gesprochenes Deutsch (DGD2, http://gd.ids-mannheim.de Schmidt et al. 2013). Diese stellt FOLK (und derzeit 18 weitere mündliche Korpora, darunter große Korpora zu binnen- und auslandsdeutschen Varietäten und weitere Gesprächskorpora, s. o.) in Form einer Website - und somit ohne größere praktische Hiirden wie die Notwendigkeit, eine spezialisierte Software zu installieren oder 
Arnulf Deppermann / Thomas Schmidt

umfangreiche Datenbestände auf den lokalen Rechner herunterzuladen - der wissenschaftlichen Öffentlichkeit kostenlos zur Verfügung.

Die DGD2 ermöglicht sowohl ein exploratives Browsen (Durchblättern) als auch ein gezieltes Durchsuchen der FOLK-Daten. In beiden Funktionalitäten wird sichergestellt, dass die Beziehungen zwischen den verschiedenen Datentypen für den Anwender unmittelbar verfüg- und nutzbar sind. So können beispielsweise aus der Darstellung eines Transkripts heraus auch Metadaten zu den beteiligten Sprechern abgerufen und zu einer gegebenen Transkriptstelle die zugehörige Stelle in der Audio-Aufnahme abgespielt werden. Ebenso bietef die Suchfunktionalität Möglichkeiten, ausgehend von jeder Fundstelle, die in der Übersicht zunächst als Keyword-In-Context (KWIC) angezeigt wird, zugehörige Sprecher- oder Ereignismetadaten, die zugehörige Transkriptstelle sowie die korrespondierende Stelle der Anfnahme abzurufen.

Auch wenn das Ziel ist, mit diesen (in Zukunft noch auszubauenden) Funktionalitäten möglichst viele Szenarien qualitativer und quantitativer Analyse zu bedienen, so erheben FOLK und die DGD2 nicht den Anspruch, die Analyseerfordernisse, die an Gesprächskorpora gestellt werden können, in ihrer ganzen Breite und vollständig abzudecken. So mögen insbesondere viele gesprächsanalytische Fragestellungen eine vertiefte ethnografische Untersuchung, verfeinerte Transkriptionen oder Annotationen notwendig machen. Die DGD2 ist daher nicht als geschlossenes System konzipiert, sondern erlaubt dem Nutzer auch, Transkriptions- und Audioausschnitte zu Einzelbelegen aus der webbasierten Umgebung heraus auf den lokalen Rechner herunterzuladen und dort mit weiteren Analyseinstrumenten (insbesondere mit einem Transkriptionseditor wie FOLKER ${ }^{1}$ oder einem Tool zur phonetischen Analyse wie Praat ${ }^{2}$ ) weiter zu bearbeiten.

\section{Verwendung von Gesprächsdatenbanken in der interaktionalen Linguistik}

Gesprächsdatenbanken lassen sich besonders im Rahmen des Paradigmas der interaktionalen Linguistik vielversprechend verwenden. Wie die Funktionalitäten der Datenbank genutzt werden können, hängt vom Typ der Fragestellung ab. Formbestimmte Fragestellungen, bei denen die Verwendung einer sprachlichen Form den Untersuchungsfokus bildet, sind ideale Kandidaten. Dies gilt für solche Formeigenschaften, die im Transkript oder durch Kodierungen und Annotate erfasst sind. Im Korpus FOLK sind dies Lautvarianten von Ausdrücken, soweit sie in der literarischen Umschrift des Transkriptionssystems GAT mithilfe des Standardalphabets repräsentiert werden können, die orthografisch normalisierten Wortformen, auf die die transkribierten Lautvarianten zu beziehen sind, und die

1 siehe http://agd.ids-mannheim.de/folker.shtml

2 siehe http://www.fon.hum.uva.nl/praat/ 
Gesprächsdatenbanken als methodisches Instrument der Interaktionalen Linguistik

Lemmata, auf die als lexikalische Nennform unterschiedliche morphologische Varianten zu beziehen sind. Alle Vorkommen von Lautvarianten, lexikalischen Formen und grammatischen Ausdrücken, die über eine dieser drei Möglichkeiten als Zeichenkette definiert werden können, können in FOLK per Suchmaschine gefunden werden. Dabei ermöglicht das Part-of-Speech-Tagging, die Suche in Bezug auf Wortarten weiter einzugrenzen.

Die maschinelle Suche ist der Ausgangspunkt fur die Erstellung einer Kollektion von Fällen. Diese wird dann anhand der Sichtung und der detaillierten Form- und Sequenzanalyse der einzelnen Fälle verfeinert (s. Abschn. 5; vgl. Deppermann 2008, Kap. 6.3). Die Möglichkeit, aufgrund von ausgewählten Metadatenparametern virtuelle Korpora zu definieren, erlaubt es außerdem, die Suche auf bestimmte Kontextbedingungen wie Sprechergruppen, Interaktionstypen oder Aufnahmeregionen zu beschränken. Es ist geplant, auch formale gesprächsstrukturelle Merkmale als Suchbedingung einbeziehen zu können, so etwa das Vorkommen einer Form vor, innerhalb oder nach einer Überlappung mehrerer Sprecher oder die Position des Phänomens am Tumanfang, in der Mitte oder am Ende des Turns (wie z. B. in der französischen Datenbank CLAPI; vgl. Groupe ICOR 2008a, b).

Weniger geeignet für maschinelle Suchen sind formal-abstrakte und interpretative Plıänomene in Gesprächen. Gemeint sind damit abstrakte, nicht-formdefinierte grammatische und semantische Klassen (wie z.B. Transitivkonstruktion, Bewegungsverb), Diskurstopiks (Sprechen über Politik) und pragmatisch-interaktionale Phänomene, die eine Interpretation des Interaktionsgeschehens erfordern (z. B. sprachliche Handlungen wie Fragen oder Beleidigungen; längere Diskursabschnitte, die kommunikative Gattungen wie Klatsch oder Belehrungen ausmachen; inferenzielle Bedeutungen wie Anspielungen oder Ironie; Phänomene der Beziehungs- und Identitätskonstitution wie $>$ Selbst-Fremdpositionerung als glaubwürdig oder >Inszenierung von epistemischer Überlegenheit<). Diese Untersuchungsgegenstände können nicht direkt als solche zum Gegenstand einer Suchanfrage gemacht werden. Dies erforderte, dass die entsprechenden Phänomene in den Daten annotiert werden. Solche Annotationen werden in FOLK nicht vorgenommen, da sie enorm aufwändig (und deshalb den Korpusausbau verlangsamend), immer nur für spezifische Forschungszwecke in einer speziellen Granularität (z.B. >Erzählung< vs. >autobiographische Erzählung< vs. >Opfererzählungs) brauchbar und manchmal auch theorieabhängig sind (z. B. >Narrations als genereller Terminus vs, >Berichten $<$ vs, >Erzählen ). Ein datenbankbasiertes Korpus wie FOLK kann dennoch zur Untersuchung solcher Phänomene benutzt werden. Zum einen bietet es die Möglichkeit, die einzelnen Gesprächstranskripte und -aufnahmen daraufhin durchzusehen, wo Fälle đẹ interessierenden Gesprächsphänomene vorkommen, und diese dann »von Hand « zu samplen und auszuwerten. Da sie oft unter besonderen Beteiligungskonstellationen interessieren, können mit Hilfe der Metadaten die potenziell interessierenden Gespräche als virtuelles Korpus eingegrenzt werden. Zum anderen gibt es manchmal typische formale Indikatoren fïr bzw. Realisierungen von interpretativ konstituierten Gesprächsphänomenen (z. B. 
Arnulf Deppermann / Thomas Schmidt

pejorative Personenbezeichnungen in Beschimpfungen, die Diskursmarker also oder $d a s$ heißt als Reformulierungsindikatoren), die man maschinell suchen kann. Solche Phänomene können den Ausgangspunkt für Beobachtungen und Hypothesen bilden, die dann anhand von einzelfallbasierten, sequenzanalytisch-interpretativen Untersuchungen weiterer Vorkommen, die aufgrund der Durchsicht und Analyse der Transkripte und Audiodaten gesucht werden müssen, geprüft und vertieft werden. Das Gleiche gilt auch für phonetische und prosodische Phänomene. Sie werden in FOLK nicht transkribiert bzw. annotiert, können aber đurch Abhören der Audiodaten erschlossen werden.

\section{Eine exemplarische Untersuchung: der Diskursmarker das heijst}

Im Folgenden zeigen wir, wie das Korpus FOLK für eine interaktionslinguistische Untersuchung genutzt werden kann. Als Beispiel wählen wir den Diskursmarker das heißßt (in Bezug auf das Französische s. Kotschi 1990). Diskursmarker sind eine häufige Erscheinung in der verbalen Interaktion. Sie sind charakteristisch für die Grammatik der gesprochenen Sprache (Schiffrin 1987; Heritage 1984; Auer/ Guinthner 2005; Fiehler et al. 2004). Mit Diskursmarkern zeigen Sprecher, in welcher pragmatischen Relation ein Tum (bzw, eine Turnkonstruktionseinheit) zu einem anderen, (meist) vorangehenden Turn (bzw. Turnkonstruktionseinheit) steht. Diskursmarker im Deutschen zeichnen sich durch ihre Stellung außerhalb des Satzrahmens (im Vorvorfeld) aus, sie sind weder in Rektionsbezichungen eingebunden noch üben sie Rektion aus. Diskursmarker sind homonym zu anderen Konnektoren, Adverbien und Adjektiven (z. B. obwohl, klar) oder deverbal (ich mein, siehste). Eine primäre Funktion von Diskursmarkern ist es, Verstehensanweisungen für das Folgende, den Turnabschnitt, der in ihrem Skopus liegt, zu geben (Fiehler et al. 2004).

Da sie anhand ihrer morphologischen Form zu suchen sind und häufig vorkommen, sind Diskursmarker ein idealer Gegenstand für eine datenbankgestützte Untersuchung. Wir nehmen in der DGD2 zunächst eine Suche nach der normalisierten Form heißt vor. Sie ergibt 630 Treffer. Da für eine interaktionslinguistische Untersuchung die Analyse jedes Einzelbelegs erforderlich ist, grenzen wir die Trefferanzahl auf eine manuell handhabbare Menge ein. Wir wählen eine Zufallsstichprobe von 200 aus den 630 gefundenen Instanzen von heißt. Zumeist, und so auch in unserem Fall, sind Suchen tiberinklusiv, d.h. sie ergeben viele falsche Positive, die gar nicht Fälle der interessierenden Form bzw. Praktik sind. Daher filtern wir aus der Zufallsstichprobe diejenigen Verwendungen von heißt aus, die nicht den Konnektor das heißt betreffen (siehe Abbildung 4). ${ }^{3}$

3 Dies sind alle Fälle, in denen heißt mit einem anderen Subjekt als das vorkommt. 
Gesprächsdatenbanken als methodisches Instrument der Interaktionalen Linguistik

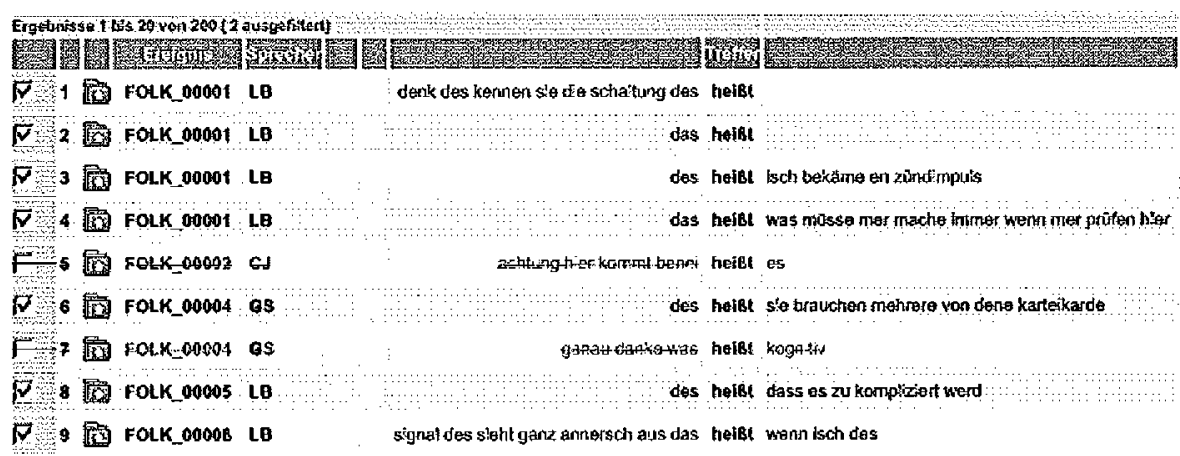

Abbildung 4: Manuell gefilterte Liste von Suchergebnissen für sheißtく

Die uns interessierende Diskursmarkerverwendung von das heißt hat die nichtreferenzielle, anaphorische Bedeutung als Reformulierungsindikator: >mit der vorangegangenen Äußerung (Wort, Phrase, Satz, längerer Turn) will der Sprecher das Folgende zum Ausdruck bringens. Dies trifft für 95 der 200 zufällig aus der Gesamtmenge der heißt-Treffer ausgewähłten Fälle zu. Dabei zeigt sich, dass die Berücksichtigung der lautlichen Formvarianten wichtig war: In 32 der 95 Fälle ist das Pronomen das als des (süddeutsche regionalsprachliche Variante) realisiert, einmal klitisiert als »s_heißt«.

Die einfache KWIC-Ansicht reicht zumeist nicht aus, um eine syntaktische Analyse der gefundenen Fälle vornehmen zu lassen. Wir müssen dazu den angezeigten Kontext der Belege auf mindestens fünf, manchmal aber auch mehr Segmente erweitern (siehe Abbildung 5).

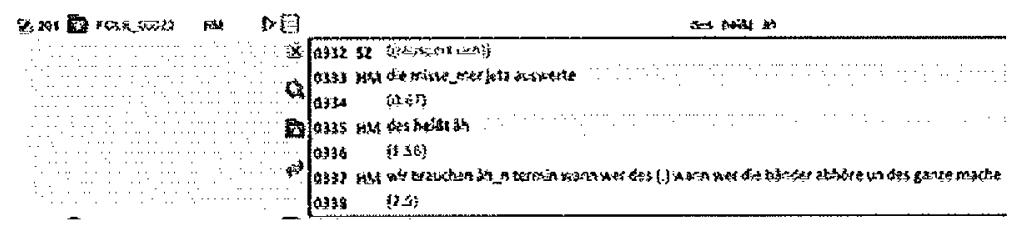

Abbildurg 5: Anzeigen des erweiterten Kontexts eines Belegs in der KWIC

Meist hat heipt dann die Semantik seinen Namen/eine Bezeichnung tragen « (z. B. "wie heißt die«), sin einer anderen Sprache bedeuten< (»weißt du was freunde auf türkisch heißt«), >die Angemessenheit einer Formulierung in Frage stellen< (walso was heißt nachteil «) oder s Sachverhalt $A$ ist Ursache für Sachverhalt $B<$ (»nur weil ich en mann bin (.) heißt des ja noch lange nich dass der mich anbaggert «). Doch auch das heijt kann in einer dieser Bedeutungen vorkommen, z.B. in $»$ das heißt (.) die selektive prüfung des steuergerătes $(0.6)$ der begriff wird ihne $(0.35)$ noch öfters äh begegnenk. 
Amulf Deppermann / Thomas Schmidt

Es ergibt sich ein klarer Befund: In 85 von 95 Fällen folgt das heißt ein syntaktisch unverbundener Hauptsatz - das heißft ist hier, wie in den Segmenten 0976 und $0984 \mathrm{im}$ folgenden Ausschnitt, Diskursmarker.

\begin{tabular}{|c|c|c|}
\hline 0972 & $\mathbf{L B}$ & [dieser] kleine messstrom (.) reischt leider nischt aus \\
\hline 0973 & & $(1.09)$ \\
\hline 0974 & LB & ja \\
\hline 0975 & & $(0.39)$ \\
\hline 0976 & LB & des heißt sie ham hier en problem \\
\hline 0977 & & $(0.68)$ \\
\hline 0978 & LB & sie haben \\
\hline 0979 & & $(0.42)$ \\
\hline 0980 & LB & er hat_s ja gsagt der herr grauberg fünf mal die null sieben \\
\hline 0981 & & $(0.27)$ \\
\hline 0982 & LB & kommt ifr messstrom nisch dursch \\
\hline 0983 & & $(0.71)$ \\
\hline 0984 & LB & des heilft ste können zunächscht mal diese sekundärspule \\
\hline 0985 & & $(0.36$ \\
\hline 0986 & L.B & sekundärmäBig nischt prüfen \\
\hline
\end{tabular}

Transkriptausschnitt 1:FOLK_E_00009_SE_01_T_01/00:20:12.81

In drei Fällen ist der folgende Satz ein Fragesatz, z. B. »das heißt was müsse mer mache immer wenn mer prüfen hier (1.06) wenn_s steuergerät was machen solk (FOLK_E_00001_SE_01 T 02/00:41:53.55 ${ }^{4}$ ). Wir sehen also, dass das heijt nicht in jedem Fall eine folgende Reformulierung ankündigt, sondern auch benutzt werden kann, um anzuzeigen, dass eine Aufforderung zu einer Reformulierung folgt.

Nur in 7 Fällen wird das heift als Matrixsatz mit folgendem abhängigen dassNebensatz verwendet. Diese Fälle haben wir auf einen Blick, wenn wir die ausgewählten Treffer nach ihrem rechten Kontext, d. h. dem Wort, das dem gesuchten Element folgt, alphabetisch sortieren (siehe Abbildung 6):

In drei Fällen wird die Matrixsatzstruktur, in keinem aber der Diskursmarker negiert, so in »ich hab jetz hier mal_n paar mögliche ergebnisse des heißt net dass des perfekt is was jetz da so dran is aber « (FOLK_E_00120_SE_01_T_02/

4 Wir zitieren Einzelbelege aus dem FOLK-Korpus in der Form FOLK_E_xxxxx_SE_01_T_yy/hh:mm:ss.ss. Dabei entspricht der erste Bestandteil der DGD2-Kennung des betreffenden Transkripts (xxxxx ist die Nummer des Gesprächsereignisses, yy die Nummer des zugehörigen Transkripts), der zweite Bestandteil gibł den Zeitpunkt in der zugehörigen Aufnahme an. Diese Form der Zitierung bleibt auch dann gültig, wenn - was von einer Version des Korpus zur nächsten der Fall sein kann - Details des Transkripts korrigiert oder ergänzt werden. 
Gesprächsđatenbanken als methodisches Instrument der Interaktionalen Linguistik

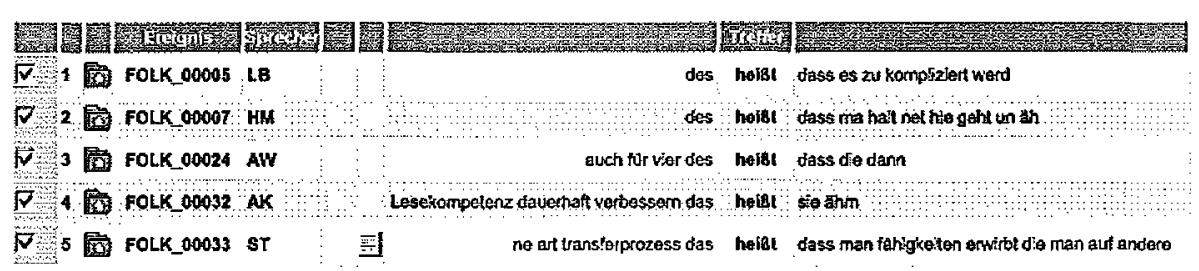

Abbildung 6: 'Treffer in der KWIC, nach rechtem Kontext sortiert

00:02:20.15). Dies ist ein weiterer Hinweis auf die grammatikalisierte und lexematisch verfestigte Natur der Diskursmarkerverwendung.

Nur in zwei der 95 Fälle von das heißt als Reformulierungsindikator folgt auf das heißt eine subsentenzielle Struktur (d. h. nur eine NP oder eine AdjP) wie hier: »als ein $[\ldots]$ größtenteils konventionell gewordenes system ${ }^{\circ} \mathrm{hh}$ das heißt sprachbewusstheit (.) und wozu is denn das wichtig « (FOLK_E_00033_SE_01_T_02/00:26:47.07). Wenn wir den Kontext uber 5 Segmente hinaus erweitern, sehen wir, dass die Sätze, die auf den Diskursmarker das heißt folgen, in 30 Fällen komplexe Sätze mit mindestens einem Nebensatz sind. Wenn wir nach dem Erreichen der maximalen Entfaltung der KWIC-Ansicht in die Volltranskripte und Aufnahmen der Belege wechseln, stellen wir außerdem fest, dass der Skopus von das heißt sich oftmals über weit mehr als den folgenden Satz erstreckt und komplexe Beschreibungen, Argumentationen und Abfolgen von Selbstreformulierungen (mehrere das heißt-Konstruktionen nacheinander) umfasst (vgl. Transkriptausschnitt 1 oben). Unsere Untersuchung legt einen interessanten Befund nahe: Es scheint eine Arbeitsteilung zwischen also und das heißt zu geben. Während nach also Reformulierungen folgen, die fast immer kürzer als ein Satz sind und komplexe Aussagengefüge (Erzählungen, Argumentationen) auf einen Begriff bringen (Deppermann 2011), verwenden Sprecher das heißt zu expandierenden Reformulierungen, die eher erläutern und komplexe Schlussfolgerungen ziehen als verbegrifflichen (vgl. a. Bührig 1996, 191 f.).

Wenn wir Treffer nach linkem bzw. rechtem Kontext sortieren, sehen wir, dass es erstaunlich wenig ausgeprägte Kookkurrenzpartner von das heißt gibt. Unmittelbar vorangehend finden wir 9 mal also. Unmittelbar folgend finden wir je 6 mal also und $\ddot{a} h(m)$. Also als Kookkurrenzpartner von das hez $\beta t$ bekräftigt weiter, dass das heißt eine inferenzielle Reformulierung ankündigt.

Das heißt kann sowohl zur Selbst- wie zur Fremdreformulierung benutzt werden. Im Korpus ibberwiegen die Selbstreformulierungen (82/95 Fälle), das heißt erscheint dann in der Mitte eines Sprecherbeitrags und wird zur Verdeutlichung oder Spezifikation der Redeintention benutzt, wie im Transkriptausschnitt 1.

In 13 der 95 Fälle zeigt das heißt eine Fremdreformulierung einer unmittelbar vorangehenden Partneräußerung an und wird als Verstehensdokumentation (vgl. Deppermann/Schmitt 2009) eingesetzt. Es wird dabei am Turnbeginn produziert: 
Arnulf Deppermann / Thomas Schmidt

$\begin{array}{lll}0064 & \text { HY } & \begin{array}{l}\text { er unternimmt zwar was aber er wartet so wie er (.) auf_m stuhl } \\ (0.25) \\ 0066\end{array} \\ 0067 & \text { HK } & \begin{array}{l}\text { ja } \\ (0.59)\end{array} \\ 0068 & & \text { und lebt au nicht sein leben richt[ig ] } \\ 0069 & \text { HY } & \text { [das heiBt] er bleibt passiv } \\ 0070 & \text { HK } & \text { ja } \\ 0071 & \text { HY } & (0.31) \\ 0072 & & \text { anstatt (.) aktiv zu werden so wie du auch gesagt hast rene (.) ja } \\ 0073 & \text { HK } & \text { Transkriptausschnitt 2: FOLK_E_00120_SE_01_T_02/00:41:52.77 }\end{array}$

Um dies festzustellen, können wir uns nicht auf die KWIC-Ansicht verlassen: Die DGD zeigt 37Fälle an, in denen vor das heißt kein linker Kontext erscheint. Dabei handelt es sich aber nicht immer um Turnanfänge. In 29 der Fälle liegen turninterne Pausen vor, die, weil sie separat transkribiert werden, dazu führen, dass sich der Turn auf mehrere Transkriptionssegmente verteilt. Da Transkriptionssegmente somit nicht Turns entsprechen und letztere nicht als solche kodiert werden, kann der Turnbeginn nicht automatisch identifiziert werden. Umgekehrt gibt es auch Fülle, in denen am Turnbeginn Vorlaufelemente vor das heißt erscheinen (»äh«, »also«), ohne dass aber die vorangehende Partneräußerung innerhalb des Turns paraphrasiert, pronominalisiert oder wiederholt wird. Entscheidend für die Verwendung als Fremdreformulierung am Turnbeginn ist also nicht der Turnbeginn im Sinne des Beginns der vokalen Turnproduktion, sondern ob eine vorangehende Partneräußerung oder ein vorangehender Teil des eigenen Turns im gleichen Turn vor das heißt reformuliert wurde.

Aufgrund dieser Befunde ist es nun möglich, unsere Kollektion weiter zu spezifizieren. Wenn wir uns beispielsweise ausschließlich für die Fälle als Fremdreformulierung interessieren, könnten wir in den aus der anfänglichen Zufallsstichprobe ausgeschlossenen 430 Fälle die weiteren Verwendungen zur Fremdreformulierung suchen und zu den bereits gefundenen 13 Fällen als Kollektion hinzufügen. Wir könnten dann zum Beispiel weiter untersuchen, in welchen Interaktionstypen und welchen Sprechern diese Verwendung vorgenommen wird.

\section{Fazit und Reflexion}

In diesem Beitrag haben wir zu zeigen versucht, wie die im Korpus FOLK verfugbaren Gesprächsdaten für interaktionslinguistische Forschungen genutzt werden können. Das Korpus FOLK und die datentechnische Infrastruktur der DGD unterstittzen die verschiedenen methodologischen Anforderungen, die sich einer in* teraktionslinguistischen Untersuchung stellen: Sie erlauben die extensive und detaillierte sequenzielle Analyse von Einzelfällen durch den Zugriff auf vollständige Transkripte und Aufnahmen, die Bildung von Kollektionen eines sprachlichen 
Gesprächsdatenbanken als methodisches Instrument der Interaktionalen Linguistik

Phänomens durch systematische, maschinell unterstitzte Suchoperationen und die Abschätzung der Bereichsspezifik und Generalisierungsreichweite von Befunden durch ausführliche Metadateninformationen über Sprecher und Gesprächsereignisse. Mithilfe der Bildung virtueller Korpora können bereichsspezifische Verwendungen identifiziert und miteinander kontrastiert werden. Die Möglichkeit, Ausschnitte von Tondaten und Transkripten zu exportieren, erlaubt dem Nutzer, einschlägige Daten weiter gemäß den eigenen Analyseinteressen aufzuarbeiten (z.B. prosodisch zu transkribieren, mit PRAAT auszumessen etc.). Das Korpus FOLK kann als alleinige Basis für eine Untersuchung benutzt oder aber als Kontrastkorpus verwendet werden, um die Spezifik bzw. Generalität der Befunde, die an einem anderen Korpus gewonnen wurden, zu testen.

Mangels hinreichend umfangreicher Korpora, der Tatsache, dass brauchbare Korpora oft nicht wissenschaftsöffentlich verfügbar sind und dass leistungsfähige Erschließungsinstrumente fehlen, wurden Gesprächsdatenbanken in der interaktionalen Linguistik (wie generell zur Untersuchung gesprochener Sprache) nur selten als Basis für Untersuchungen verwendet. Hinzu kommen die für die qualitative Forschung typischen, methodologischen Vorbehalte gegen die Untersuchung von nicht vom Forscher selbst erhobenen Daten, die Brauchbarkeit maschineller Instrumente der Korpuserschließung, welche eine formale und melsr oder weniger kontextfreie Definition von zu suchenden Strukturen erfordern, und die Quantifizierung von Befunden. Dies sind u. E. keineswegs grundsätzliche Einwände gegen die Verwendung von Gesprächsdatenbanken als Datengrundlage und methodisches Hilfsmittel, sondern vielmehr Hinweise auf Probleme, Aufgaben und methodische Vorsichtsmaßnahmen, die im Forschungsprozess zu beachten sind. Wir haben verschiedentlich darauf hingewiesen, dass maschinell gewonnene Suchergebnisse, in bestimmten Ansichten bereitgestellte Repräsentationen der Daten und statistische Kennwerte auf keinen Fall unbesehen als Grundlage linguistischer Erkenntnisse genommen werden können und đass sie bei naiver Nutzung zu Aussagen führen, die Methodenartefakte sind. Auch sind reine Verteilungsaussagen keine linguistisch relevanten Erkenntnisse, sondern nur Befunde, die interpretiert und erklärt werden müssen. Maschinelle Suchen helfen die Korpuserschließung zu systematisieren. Sie ersetzen aber nicht den Zugang zu den Primärdaten, die detaillierte linguistische Analyse und die Interpretation der Daten; dies umso mehr, je interpretativer das Untersuchungsphänomen ist.

Wenn eine Gesprächsdatenbank entsprechend methodologisch reflektiert eingesetzt wird, bietet sie u. E. unschätzbare, noch kaum genutzte Erkenntnismöglichkeiten, die mit kleineren Korpora und ohne maschinelle ErschlieBungsinstrumente nicht zu haben sind. Unsere kleine exemplarische Anwendung hat gezeigt, dass eine Gesprächsdatenbank wie FOLK zum einen die induktive Entdeckung von Mustern und Verteilungen (in Statistiken, KWIC-Ansichten, Sortierungen kookkurrenter Kontexte) unterstützt. Gerade der größere Umfang und die breitere Stratifikation von FOLK im Vergleich zu den viel kleineren ForschungsprojektKorpora lässt Muster hervortreten, die in kleinen Samples und erst recht in Ein- 
Atnulf Deppermann / Thomas Schmidt

zelfallanalysen unbemerkt bleiben, über deren Systematizität und deren Abhängigkeit von spezifischen Kontextbedingungen nicht entschieden werden kann. Genauso unterstützt FOLK aber auch die Varianten- und Bedingungssuche und das Testen von Hypothesen durch die Bereitstellung aussagekräftiger Datenmengen und die Möglichkeit systematischer, maschinell unterstützter Korpuserschließung. Im Forschungsprozess verbinden sich induktive und deduktiv-testende Erkenntnisbildung in einer Bewegung der zunehmenden Verfeinerung von Hypothesen und der korrespondierenden Belegkollektionen. Das Ziel besteht dann in einer Darstellung sprachlicher Praktiken, die sowohl ihrer kontextsensitiven Adaptivität als auch ihren generischen, kontextfreien bzw. -typspezifischen Potenzialen gerecht wird (vgl. Heritage 2010) und dabei das Generalisierungsniveau der wissenschaftlichen Aussagen korpusbasiert empirisch fundiert.

\section{Literatur}

Auer, Peter/Günthner, Susanne: Die Entstehung von Diskursmarkern im Deutschen ein Fall von Grammatikalisierung? In: Grammatikalisierung im Deutschen. $\mathrm{Hg}$. von Torsten Leuschner und Tanja Mortelsmans. Berlin 2005, S. 335-362.

Bethge, Wolfgang/Bonnin, Gunther M.: Proben deutscher Mundarten. $123 \mathrm{~S}$. und 1 Karte. Täbingen 1969.

Berens, Franz-Josef u.a.: Projekt Dialogstrukturen. Ein Arbeitsbericht. Heutiges Deutsch I/12. München 1976.

Bührig, Kristin: Reformulierende Handlungen. Zur Analyse sprachlicher Adaptierungsprozesse in institutioneller Kommunikation. Tübingen 1996.

Deppermann, Arnulf: Gespräche analysieren. Wiesbaden 2008.

Deppermann, Arnulf: Notionalizations: The transformation of descriptions into categorizations. In: Human Studies 34, 2, (2011), S, 155-181.

Deppermann, Arnulf/Hartung, Martin: Was gehört in ein nationales Gesprächskorpus? Kriterien, Probleme und Prioritäten der Stratifikation des $\gg$ Forschungs- und Lehrkorpus Gesprochenes Deutsch« (FOLK) am Institut für Deutsche Sprache (Mannheim). In: Korpuspragmatik. Hg. von Ekkehard Felder, Marcus Müller und Friedemann Vogel. Berlin, S. 414 450.

Deppermann, Arnulf/Schmitt, Reinhold: Verstehenstokumentation: Zur Phänomenologie von Verstehen in der Interaktion. In: Deutsche Sprache 36, 3, (2009), S. 220-245.

Fandrych, Christian/MeiBner, Cordula/Slavcheva, Adriana: The GeWiss Corpus: Comparing Spoken Academic German, English and Polish. In: Multilingual corpora and multilingual corpus analysis. Hg. von Thomas Schmidt und Kai Wörner. Amsterdam 2012, S. 319-337.

Fiehler, Reinhard u. a.: Eigenschaften gesprochener Sprache. Theoretische und empirische Untersuchungen zur Spezifik mündlicher Kommunikation. Tübingen 2004.

Groupe ICOR (M. Bert, S. Bruxelles, C.Etienne, L. Mondada, V. Traverso): Tool-assisted analysis of interactional corpora: »voila» in the CLAPI database. In: Journal of French Language Studies 18 (2008), S. 121-145. 
Gesprächsdatenbanken als methodisches Instrument der Interaktionalen Linguistik

Groupe ICOR ( M. Bert, S. Bruxelles, C. Etienne, L. Mondada, S. Teston, V. Traverso): soh::, oh là là, oh ben... $<$ : les usages du marqueur »oh « en français parlé en interaction. In: Actes du Congrès Mondial de Linguistique Française (CMLF08). Hg. von Durand, J., Habert, B., Laks, B. Paris 2008, 17 pages (CD-ROM). ( DOI: 10.1051/cmlf08099).

Heritage, John: A change-of-state token and aspects of its sequential placement. In: Structures of Social Action. Hg, von John M. Atkinson und John Heritage. Cambridge 1984 , S. $299-345$.

Heritage, John: Conversation Analysis: Practices and Methods. In: Qualitative Sociology (3rd Edition). Hg. von David Silverman. London 2010, S. 208-230.

Hinrichs, Erhard et al.: The Tübingen Treebanks for Spoken German, English, and Japanese. In: Verbmobil: Foundations of Speech-to-Speech-Translation. Hg. von Wolfgang Wahlster. Berlin 2000, S. 552-576.

Kotschi, Thomas: Reformulierungshandlungen und Textstruktur. Untersuchungen zu frz. c'est-a-dire. In: Sprache und Pragmatik 19 (1990), S. 1-27.

Labov, William: Sociolinguistic Patterns. Philadelphia 1972.

Wiese, Heike: Kiezdeutsch. Ein neuer Dialekt entsteht. München 2012.

Schiffrin, Deborah: Discourse Markers. Cambridge 1987.

Selting, Margret u. a.: Gesprächsanalytisches Transkriptionssystem 2 (GAT 2). In: Gesprächsforschung-Online-Zeitschrift zur verbalen Interaktion 10 (2009), S. 353-402.

Schmidt, Thomas/Schütte, Wilfried: FOLKER: An Annotation Tool for Efficient Transcription of Natural, Multi-party Interaction. In: Proceedings of the Seventh conference on International Language Resources and Evaluation (LREC'10). $\mathrm{Hg}$. von Nicoletta Calzolari et al. Valletta, Malta 2010.

Schmidt, Thomas/Dickgießer, Sylvia/Gasch, Joachim: Die Datenbank für Gesprochenes Deutsch - DGD2. Mannheim 2013. URN: urn:nbn:de:bsz:mh39-12747.

Steger, Hugo/Engel, Ulrich/Moser, Hugo (Hgg.): Texte gesprochener deutscher Standardsprache I. Erarbeitet vom Institut für deutsche Sprache, Forschungsstelle Freiburg. München 1971.

Westpfahl, Swantje/Schmidt, Thomas: POS für(s) FOLK- Part of Speech Tagging des Forschungs- und Lehrkorpus Gesprochenes Deutsch. In: Journal for Language Technology and Computational Linguistics, erscheint 2014.

http://agd.ids-mannheim.de/folker.shtml [letzter Zugriff 1/14].

http://dgs.ids-mannheim.de [letzter Zugriff 1/14].

http://www.fon.hum.uva.nl/praat/ [letzter Zugriff 1/14].

Prof. Dr. Arnulf Deppermann, Institut für Deutsche Sprache Mannheim, deppermann@ids-mannheim.de

Dr. Thomas Schmidt, Institut für Deutsche Sprache Mannheim, thomas.schmidt@idsmannheim.de 